\title{
Story Validation and Approximate Path Inference with a Sparse Network of Heterogeneous Sensors
}

\author{
Jingjin $\mathrm{Yu}$ \\ jyu18@uiuc.edu \\ Department of Electrical and Computer Engineering \\ University of Illinois \\ Urbana, IL 61801 USA
}

\author{
Steven M. LaValle \\ lavalle@uiuc.edu \\ Department of Computer Science \\ University of Illinois \\ Urbana, IL 61801 USA
}

\begin{abstract}
Given a story from an agent (sensor outputs from a robot or a tale told by a human) and recordings from a spare network of heterogeneous sensors, this paper provides efficient algorithms that validate whether it is possible to reconstruct a path compatible with the sensor recordings that is also "close" to the agent's story. In solving the proposed problems, we show that effective exploitation of a unique finite automaton structure yields time complexity linear in both the length of the story and the length of the sensor observation history. Besides immediate applicability towards security and forensics problems, the idea of behavior validation using external sensors also appears promising in complementing design time model verification.
\end{abstract}

\section{INTRODUCTION}

Surveillance cameras at supermarkets. Occupancy sensors in office rooms. Buried pressure based vehicle sensors at traffic lights. Your thermostat at home. Sensors are everywhere: Due to diminished cost, an ever increasing number of heterogeneous, more reliable sensors are being installed and many are also networked. Some of these sensors are part of smart systems, such as automatic traffic citation issuing systems using cameras installed at cross sections. However, this vast network of sensors is far from realizing its full potential since the integration of sensor data usually requires human intervention. For example, video surveillance systems often merely fuse images from different cameras for human interpretation, which can be error prone. More effective use of networked sensors then calls for the development of specialized and efficient algorithms for these systems.

With the research presented here, we hope to bring us one step closer to the goal of maximizing the utility of networked sensor systems. In [33], we introduced and solved a problem in which an agent's account (a story) of its path in an environment is validated against recordings from a sparse network of sensors deployed in the same environment. In that work, an agent's story is required to be error-free and has start/end time matching those of the sensor recordings'. Such formulations are restrictive for two reasons: 1. Even a truthful agent is likely to introduce errors in recalling a long story, especially when the agent in question is a human; 2. Requiring matching start/end time can be hard to guarantee. Moreover, when an agent's story is not consistent with an observation history, the algorithms from [33] do not provide an alternative path for the agent that is "close" to the agent's story. In this paper, we provide highly efficient algorithmic solutions to address and remove all these limitations.

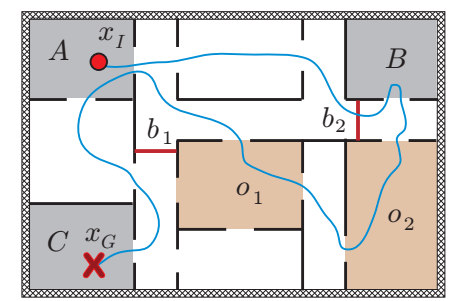

Fig. 1. A workspace with rooms $A, B, C$, occupancy sensors $o_{1}, o_{2}$ and beam detectors $b_{1}, b_{2}$. The curve connecting the start (circle) and goal (cross) locations is a possible agent path for the story $A, B, A, C$, which triggers the sensor recordings $b_{2}, o_{2}, o_{1}$, in that order.

Our study also complements the verification of robotic system design. From the perspective of modeling, a robotic agent in our problem can be viewed as a hybrid system: It goes from room to room to carry out tasks, changing its operation modes along the way. Since the verification of an autonomous system with continuous state space and control input is undecidable in general [2], it is desirable to have external measures to keep in check the unverified portion of such systems. Even for hybrid systems with provably correct designs, such as autonomous robots or cars [8], [15] based on specifying high level tasks using General Reactivity(1) formulas [22], a malfunction could occur due to sensor/actuator/computer errors. Furthermore, complex hybrid systems are also subject to deliberate tempering to behave in unwanted manners. Therefore, automatically monitoring hybrid systems with external sensors can be an effective error correcting and safeguarding measure.

Sensor networks based approaches have been applied to infer basic properties of moving bodies in its range. Networks of binary proximity sensors have been employed to track one or multiple moving bodies using various analytical tools [3], [14], [25], [26]. The task of counting multiple targets is also studied under different assumptions [4], [13]. In these works, the sensors' aggregate sensing range must cover the target(s) at all times, which we do not assume. When only subsets of an environment are guarded, word problems in groups [9], [12] naturally arise. For the setup in which targets moving inside a $2 \mathrm{D}$ region are monitored with a 
set of detection beams, [28] characterizes possible target locations, target path reconstruction up to homotopy, and path winding numbers. In this domain, the surfacing of more interesting behaviors also induces an increase in complexity; few efficient algorithms exist.

In obtaining solutions to more general problems, it becomes clear that the story validation problem we raise can be transformed to the string edit distance problem between a string and a regular language, with first algorithmic solution appearing in [30]. Improvements on time and space requirements for algorithms solving such problems can be found in [31], [24], [19], [1]. The best general algorithm for obtaining a string with smallest edit distance from a string $x$ between $x$ and an automaton $A$ appears to be the one given in [1], which takes time $O\left(|x||A| \lg |A|_{Q}\right)(|A|$ is the sum of the number of states and transitions of $A$ and $|A|_{Q}$ is the number of states of $A$ ). For an overview of approximate string matching problems, see [20]. In viewing the resemblance of our problem to the questions asked in [3], [25], [28], our solution follows an information space approach [16], which requires the design of a combinatorial filter, similar to those in [17], [29], [32]. These combinatorial filters are minimalist counterparts to widely known Bayesian filters [5], [7], [11], [18], [21], [27].

The rest of the paper is organized as follows. Section II provides formulations of the three problems we study in this paper. Section III briefly reviews the algorithmic solution to the base problem and apply it to solve the first of the three problems. Section IV relates our problem to the string edit distance problem. We then combine the gained insight with our special problem structure to provide algorithms for solving the other two problems with further efficiency gains, in Section V. We also discuss additional extensions in this section and conclude with Section VI.

\section{Problem Formulation}

\section{A. Workspace, Agent Stories, and Observation History}

Let the workspace $W \subset \mathbb{R}^{2}$ be a bounded, path connected open set with a polygonal boundary, $\partial W$. Let one or more point agents move around in $W$, carrying out unknown tasks. Every agent has a map of $W$ and may move arbitrarily fast along some continuous path $\tau:\left[t_{0}, t_{f}\right] \rightarrow W$. We are interested in a particular agent $x$ which can be thought of as a suspect. Agent $x$ provides a story, which is a sequence of locations it recalls along its path in increasing chronological order,

$$
\mathbf{p}=\left(p_{1}, p_{2}, \ldots, p_{n}\right), \quad p_{i} \subset W .
$$

Since $p_{i}$ 's can be viewed as letters from an alphabet, $\mathbf{p}$ can be viewed as a string $p_{1} \ldots p_{n}$ as well; we use the string notation mostly in this paper since it is shorter. We assume that the unique elements of $\mathbf{p}$ are each a simply connected region with a polygonal boundary and pairwise disjoint. The set of all unique elements of $\mathbf{p}$ is denoted $\mathcal{C}_{p}$, which can be thought of as a set of rooms in $W$. As an example, for the environment from Fig. 1, agent $x$ may simply report that "I went from room $A$ to room $B$, then came back to room $A$, and eventually arrived room $C$, at which point I stopped." To limit the number of questions that can be posed, we require that agent $x$ starts from $p_{1}$ and ends in $p_{n}$. Unless otherwise stated in a particular problem, it is assumed that agent $x$ recalls in $\mathbf{p}$ locations on its path correctly.

Let a subset of the workspace $W$ be guarded by a set of occupancy sensors and beam detectors. The placement of sensors in $W$ is unknown to the agents. An occupancy sensor $o_{i}$ is assumed to detect the presence of an agent in a fixed, convex subset $s \subset W$. For example, a room may be monitored by such a sensor $\left(o_{1}, o_{2}\right.$ in Fig. 1). A data point recorded by $o_{i}$ has two parts, an activation, $r_{o a}=\left(o_{i}, t_{a}\right)$, and a deactivation, $r_{o d}=\left(o_{i}, t_{d}\right)$. Here $t_{a}$ is the time when the first agent enters an empty $s$ and $t_{d}$ is the time when the last agent exits $s$. A beam detector $b_{i}$, on the other hand, guards a straight line segment, $\ell \subset W$, between two edges of $\partial W\left(b_{1}, b_{2}\right.$ in Fig. 1). A data point of such a sensor is recorded as an agent crosses $\ell$, which can be represented by a 2-tuple, $r_{b}=\left(b_{i}, t\right)$. A beam detector is deactivated right after activation. We further assume that when a beam detector is triggered by an agent, the agent must pass from one side of the beam to the other side.

If we gather all sensor recordings (of the types $r_{o a}, r_{o d}, r_{b}$ ) during a time interval $\left[t_{0}, t_{f}\right]$ and order them by time incrementally, an observation history is obtained. This sequence is unique since it is reasonable to assume that no two recordings happen at the exact same time. As we do not assume that an agent provides the exact time when it visits a location, the time in the sensor recording is also relative. Therefore, when we compose the observation history of the sensors, we may discard the time element of each sensor recording, keeping only their relative order. A simplified observation history can be written as (each $s_{i}$ corresponds to the region covered by a sensor):

$$
\mathbf{s}=\left(s_{1}, s_{2}, \ldots, s_{m}\right), \quad s_{j} \subset W .
$$

Similar to $\mathbf{p}$, we can write $\mathbf{s}$ as a string. Note that $m, n$ are of comparable size since the more places an agent goes, the more sensor recordings it triggers; the relationship between $m, n$ is linear intuitively. Also, for an occupancy sensor, there should always be an even number of appearances of it in $\mathbf{s}$, with the odd numbered appearances being activation and the even numbered appearances being deactivation. If there is a single agent in the workspace, an activation must also be followed by a deactivation.

In the example given in Fig. 1, a sensor recording of an occupancy sensor could imply that the agent enters and exits from any of the doorways; there are four doorways for $o_{1}$ and two for $o_{2}$. Similarly, when the beam detector $b_{2}$ is triggered, an agent could be passing it from left to right or in the other direction. These sensors certainly cannot distinguish among different agents. Since we work with these weak sensors, the algorithms in this paper apply to a wider range of sensors, provided that they are at least as powerful. We denote the unique sensor regions from $\mathbf{s}$ as $\mathcal{C}_{s}$. As justified in [33], we assume that any two elements of $\mathcal{C}_{p} \cup \mathcal{C}_{s}$ are disjoint (in $W$ ). 


\section{B. Validation Problems}

Given the above setup, we want to know whether a story is consistent with a sensor observation history. For example, if an agent starts from $A$ in the workspace from Fig. 1, it cannot end up at $B$ without triggering any sensor recordings. Our earlier work addressed the problem of validating a single agent's story against the observation history happening during the same time interval, in the presence of no other agent or multiple (an unknown number of) agents in the same workspace. We also assume that the agent's story is exact: The suspect agent $x$ cannot have error its description of a story. The problem of verifying an agent's story of a single agent workspace is given below, which is included here for reviewing the baseline algorithm:

Problem 1 (Exact Story, Matching Time Intervals) Let there be a single agent in a workspace. The agent provides a story $\mathbf{p}$ for the time interval $\left[t_{0}, t_{f}\right]$. During the same time interval, the sensors in the workspace produce an observation history, $\mathbf{s}$. Validate whether $\mathbf{p}$ is consistent with s. Extract a feasible path if the story is consistent.

The formulation of Problem 1 is quite restrictive in at least two ways. First, requiring the story and the observation history to span the exact same time interval $\left[t_{0}, t_{f}\right]$ may not be always possible. For example, the sensor network may be inactive at some point due to daily operation schedule or system maintenance. We capture and generalize this case with the following problem:

\section{Problem 2 (Exact Story, Mismatching Time Intervals)}

Let there be a single agent in a workspace. The agent provides a story $\mathbf{p}$ for the time interval $\left[t_{0}, t_{f}\right]$. The sensors in the workspace produce an observation history, s, for a time interval $\left[t_{0}^{\prime}, t_{f}^{\prime}\right]$ that overlaps with $\left[t_{0}, t_{f}\right]$. Validate whether $\mathbf{p}$ is consistent with $\mathbf{~}$. Extract a feasible path if the story is consistent.

The second restriction is that a truthful agent, be it human or robot, may inevitably make mistakes in recalling a story. One very likely scenario here is that the agent may have missed in $\mathbf{p}$ locations in $\mathcal{C}_{p}$ it has visited. For instance, a truthful agent may have recalled a story $\{A, B, C\}$ although its story is actually $\{A, B, A, C\}$. This scenario translates into the formulation below (For two strings/sequences $\mathbf{p}, \mathbf{p}^{\prime}$, the expression $\mathbf{p} \leq \mathbf{p}^{\prime}$ or $\mathbf{p}^{\prime} \geq \mathbf{p}$ denotes that $\mathbf{p}$ is a subsequence of $\mathbf{p}^{\prime}$ ):

\section{Problem 3 (Partial Story, Matching Time Intervals)}

Let there be a single agent in a workspace. The agent provides a story $\mathbf{p}$ for the time interval $\left[t_{0}, t_{f}\right]$. During the same time interval, the sensors in the workspace produce an observation history, $\mathbf{s}$. Let $\mathbf{p}^{\prime} \geq \mathbf{p}$ be a sequence with elements from $\mathcal{C}_{p}$. Validate whether there exists a $\mathbf{p}^{\prime}$ that is consistent with $\mathbf{s}$. If such a story $\mathbf{p}^{\prime}$ exists, find one of shortest length.
Problem 3 motivates a more general case: The agent, even with best effort, may add locations it has not visited (insertion), miss locations it has visited (deletion), or report some locations it has visited incorrectly (substitution). As an example, a truthful agent may have recalled $\{A, B, C\}$ when the actual story is $\{C, B, B, D\}$. Calling each of the three possibilities to introduce an error as an edit, we obtain the following problem:

\section{Problem 4 (Error in Story, Matching Time Intervals)}

Let there be a single agent in a workspace. The agent provides a story $\mathbf{p}$ for the time interval $\left[t_{0}, t_{f}\right]$. During the same time interval, the sensors in the workspace produce an observation history, $\mathbf{s}$. Let $\mathbf{p}^{\prime}$ be a sequence with elements from $\mathcal{C}_{p}$. Find a $\mathbf{p}^{\prime}$ that is consistent with $\mathbf{s}$ such that $\mathbf{p}$ can be obtained from $\mathbf{p}^{\prime}$ with the least number of edits.

We point out that besides the above problems, many other interesting formulations are possible. For example, one may require the suspect's story have a maximum error rate of $10 \%$, for which a solution can be derived from the solutions of Problem 4. Due to limited space, we focus on the general cases given in Problem 2 through 4; additional extensions are then discussed briefly.

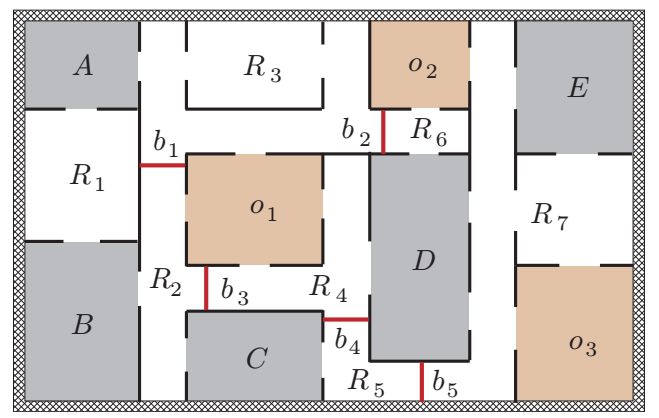

Fig. 2. A workspace with five beam detectors $b_{1}$ through $b_{5}$, three occupancy sensors $O_{1}$ through $o_{3}$, and five labeled rooms $A$ through $E$. Thus, $\mathcal{C}_{p}=\{A, B, C, D, E\}, \mathcal{C}_{s}=\left\{b_{1}, b_{2}, b_{3}, b_{4}, b_{5}, o_{1}, o_{2}, o_{3}\right\}$. There are seven connected components $R_{1}$ through $R_{7}$ when regions guarded by sensors and rooms are treated as workspace obstacles.

\section{BASEline Algorithm AND THE CASE OF Mismatching Time InTERVALS}

In this section we review the baseline algorithm for Problem 1, with the example setup from Fig. 2. Only the most essential elements from [33] are reproduced here; readers may check that paper for a more thorough explanation. After the recapitulation, we apply the algorithm to solve Problem 2.

\section{A. Baseline Algorithm}

It is observed that, when regions covered by sensors and rooms are not occupied, they act as obstacles. In the example, these obstacles partition the workspace into seven connected components, $R_{1}$ through $R_{7}$. Assuming that the workspace, rooms, and sensors are given to us as edge lists, we apply a cell decomposition procedure [6] to extract these connected 
components, which is then transformed into a graph structure that captures the connectivity of the rooms and sensors. We call this graph $G$ the connectivity graph, which extracts all necessary information needed for validating an agent's story. The connectivity graph for our example is given in Fig. 3. Each beam detector has two vertices in the graph to represent its two sides; these are marked differently from other vertices. To avoid making the graph too complicated for viewing, some of the edges are collapsed and self loops of vertices from $\mathcal{C}_{p}$ are not shown (An agent can always go out of a room and come right back without triggering any sensor recordings).

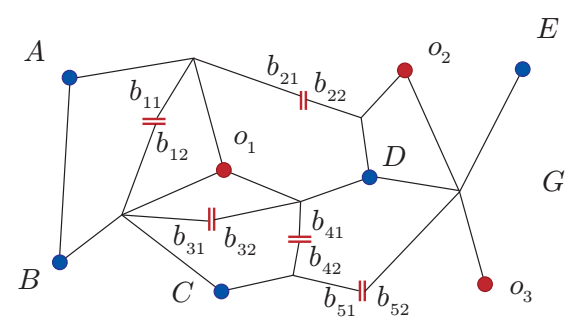

Fig. 3. The connectivity graph $G$ for the example given in Fig. 2 .

With the construction of the connectivity graph $G$, Problem 1 effectively becomes graph search. Note that at any given moment, only part of $G$ is available, depending on where the agent is and which sensor is active. Although Fig. 3 is shown as a single graph, searching through it using breath first or depth first queuing leads to exponential number of branchings with respect to the length of $\mathrm{s}$ due to beam detectors. Therefore, we need to explore additional structure in the problem. For story $\mathbf{p}=p_{1} \ldots p_{n}$ and observation history $\mathbf{s}=s_{1} \ldots s_{m}$, since the search must march through both strings forward, a position in the two strings and a location in the graph $G$ define a subproblem that is just the same as the initial problem. For example, a subproblem may be validating $p_{i} \ldots p_{n}$ against $s_{j} \ldots s_{m}$ starting from room $E$. There is clearly only a polynomial number of subproblems; if the time it takes to go from one subproblem to a smaller one is also polynomial, then the overall search is efficient in input size.

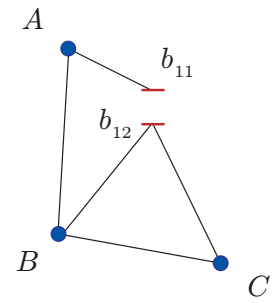

(a)

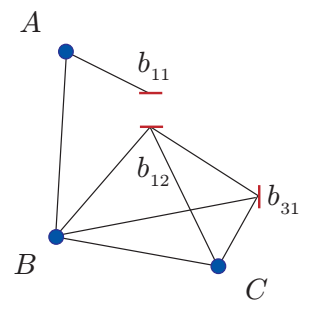

(b)
Fig. 4. a) The available subgraph of the example from Fig. 2 when $b_{1}$ is the first sensor recording. b) The available subgraph of the example from Fig. 2 for the recording $b_{1} b_{3}$.

It turns out that this is indeed the case for Problem 1. For illustration, suppose that the story is $\mathbf{p}=A B D E C$ and the sensor observation history is $\mathbf{s}=b_{1} b_{3} O_{2} o_{2} b_{4}$. First, we construct a graph based on $G$ and $\mathbf{s}$ that captures all possible agent paths for a fixed s. Since agent $x$ starts at $A$ and must first pass $b_{1}$, before $b_{1}$ is triggered, the part of $G$ that is available is $G_{1}$ (see Fig. 4(a)). Similarly, the next part of $G$ available, after agent $x$ 's passing of $b_{1}$ but not $b_{3}$, is $G_{2}$ (see Fig. 4(b)). For all such $G_{j}$ 's, agent $x$ must pass through them sequentially. Observing this, $G_{j}$ 's are chained together to get a composite graph $G_{s}$ as illustrated in Fig. 5, in which the directed edges allow only one-way passages.

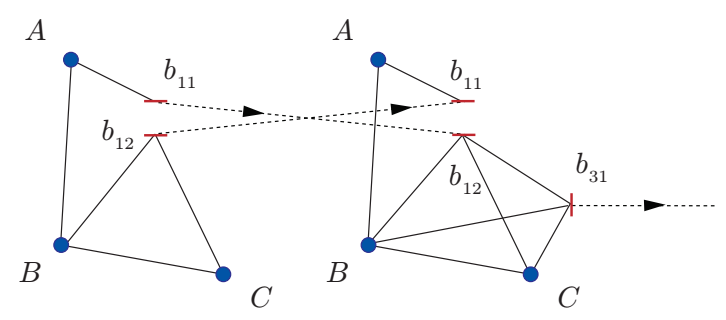

Fig. 5. Part of the composite graph that agent $x$ must pass through.

The rest of the algorithm is dynamic programming much like Dijkstra's algorithm[10]: We recurse over both $\mathbf{p}$ and $G_{s}$. More specifically, for each $p_{i}$ of $\mathbf{p}$, we mark in $G_{s}$ the possible vertices that agent $x$ can be at after going through $p_{1} \ldots p_{i}$. Even though $G_{s}$ has up to $m n_{w}$ (Let $n_{w}$ be the size of input $\left.W_{\text {free }}\right)$ vertices, there are at most $m+1$ vertices corresponding to $p_{i}$, one in each $G_{j}, j=1, \ldots, m+1$. For $p_{i}$ in $G_{j}$ (if there is one), we may denote it as $\left(p_{i}, j\right)$. We are left with deciding the total time needed for finding all $\left\{\left(p_{i}, j\right)\right\}$ reachable from $\left\{\left(p_{i-1}, j\right)\right\}$ via 1 -hop(Passing a single element of $\mathcal{C}_{p}$ ). This is not simply checking neighbors for each $\left(p_{i-1}, j\right)$ with fixed $i$, since passing a sensor vertex is a 0-hop. Here we use the special structure of $G_{s}$ again. Since $\left(p_{i}, j\right)$ belongs to $G_{j}$, it can be reached in only two ways: From the start vertices of $G_{j}$ or from $\left(p_{i-1}, j\right)$. This insight yields again a dynamic programming subroutine, with the cost of $O\left(m \lg n_{w}\right)$ per $p_{i}$. The total complexity for searching $G_{s}$ is then $O\left(n m \lg n_{w}\right)$.

The pseudocode is summarized in Algorithm 1. The subroutine BUILDCONNGRAPH $\left(W_{\text {free }}\right)$ returns the connectivity graph $G$ given an edge list representation of $W_{\text {free. }}$. The subroutine $\operatorname{SuBG}\left(G, v_{1}, v_{2}\right)$ returns the available part of $G$ starting from $v_{1}$ and ending at $v_{2}$. Finally, CHAIN $(\ldots)$ connects all input graphs sequentially. The time spent on these portion of the algorithm is $O\left(m n_{w} \lg n_{w}+n_{w}^{2}\right)$ [33]. If VAlidateAgentSTORY returns true, which means $\mathbf{p}$ is consistent with $\mathbf{s}$, a possible path can be retrieved via backtracking the search process. We now apply Algorithm 1 to Problem 2.

\section{B. Story and Observation History with Mismatching Time Intervals}

Problem 2 has several subcases, depending on how $\left[t_{0}, t_{f}\right]$ and $\left[t_{0}^{\prime}, t_{f}^{\prime}\right]$ compare. Assuming that $t_{0}, t_{f}, t_{0}^{\prime}, t_{f}^{\prime}$ are pairwise 


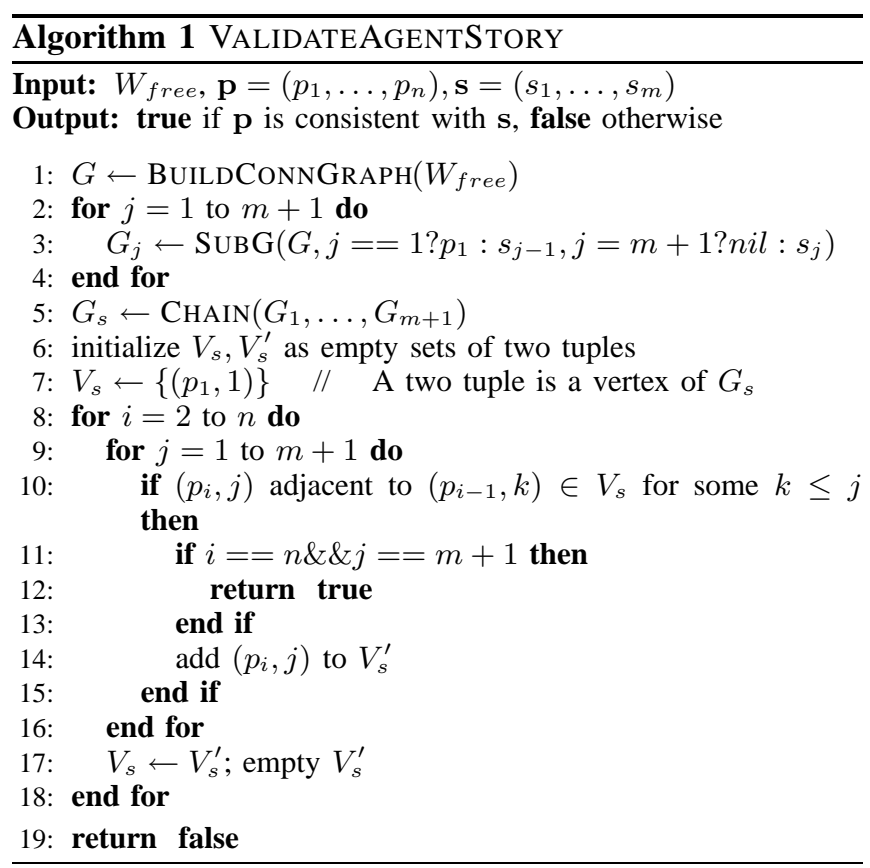

different, the following six cases are possible:

$$
\begin{array}{ll}
t_{0}<t_{f}<t_{0}^{\prime}<t_{f}^{\prime}, & t_{0}<t_{0}^{\prime}<t_{f}<t_{f}^{\prime}, \\
t_{0}^{\prime}<t_{0}<t_{f}<t_{f}^{\prime}, & t_{0}<t_{0}^{\prime}<t_{f}^{\prime}<t_{f}, \\
t_{0}^{\prime}<t_{0}<t_{f}^{\prime}<t_{f}, & t_{0}^{\prime}<t_{f}^{\prime}<t_{0}<t_{f} .
\end{array}
$$

For the first and last cases, $\left[t_{0}, t_{f}\right]$ and $\left[t_{0}^{\prime}, t_{f}^{\prime}\right]$ are disjoint. In these cases, the sensors cannot possibly observe the agent during $\left[t_{0}, t_{f}\right]$; nothing needs to be done about $\mathbf{p}$ (as long as $\mathbf{p}$ does not conflict with itself). We are not yet done, because we still need to check whether an empty story is consistent with s. This can be done using VAlidateAgEnTSTORY (The search portion only takes $O\left(m \lg n_{w}\right)$ time).

The second case is $t_{0}<t_{0}^{\prime}<t_{f}<t_{f}^{\prime}$, which means that a later portion of agent story overlaps with an earlier portion of the sensor observation history. This can be handled by running VALIDATEAGENTSTORY algorithm $n$ times. For the $i$-th run, the story is $\left(p_{i}, \ldots, p_{n}\right)$ and the sensor observation history is s. If any of the runs returns true, then the story is valid; otherwise the story is inconsistent. We note that the search can be arranged more efficiently by working on the same $p_{i}$ 's of different runs at the same time; thus, the time complexity for this case remains $O\left(n m \lg n_{w}\right)$.

For the third case, $t_{0}^{\prime}<t_{0}<t_{f}<t_{f}^{\prime}$, the story $\mathbf{p}$ may start in the middle of $G_{s}$. VALIDATEAGENTSTORY can be easily modified to handle this: Instead of starting in $G_{1}$, we now allow the search to start at $\left(p_{1}, j\right)$ for all applicable $j$ 's. If $p_{n}$ is ever reached in the search, the modified algorithm should report that $\mathbf{p}$ is consistent with $\mathbf{s}$. The time complexity again remains the same. Following along the same lines, we can decide cases four and five.

\section{The Composite Automaton Structure}

Although solution to Problem 2 is a relatively straightforward extension of the VALIDATEAGENTSTORY algorithm, it is not immediately clear whether the approach applies to Problem 3, 4, and more general cases. Part of the difficulty comes from the composite graph $G_{s}$ : It appears to have more structure than a standard directed graph with $O\left(m n_{w}\right)$ vertices. On the other hand, we observe that VALIDATEAGENTSTORY operates much like an automaton in the sense that it processes $\mathbf{p}$ sequentially and either accepts or rejects. This prompts us to ask: Can we turn $G_{s}$ into an automaton and apply results from Automata Theory to tackle our problem? We answer the first part of this question in this section and delay the second part to the next.

The conversion of a connectivity graph $G$ and a observation history $\mathbf{s}$ into finite automata is relatively straightforward. For each pair of consecutive sensor recordings $s_{i}, s_{i+1}$ (except when $i=0, m$ ), we isolate the part of $G$ that can reach vertices of $s_{i+1}$ from vertices of $s_{i}$ and convert it to an automaton. When $i=0$, we let the start state of the automaton transit to all vertices in $\mathcal{C}_{p}$ and when $i=m$, we let all vertices in $\mathcal{C}_{p}$ be acceptance states. These automata are then chained together to give a composite automaton.

As a concrete example, we assume again that the observation history is $\mathbf{s}=b_{1} b_{3} O_{2} O_{2} b_{4}$. The first sensor recording is $b_{1}$, which means that agent $x$ must at one point reach either $b_{11}$ or $b_{12}$ and cross the beam detector. From Fig. 3 it is clear that if agent $x$ starts from $D$ or $E$, it is impossible for the agent to get to $b_{1}$ without triggering other sensors. Therefore, we only need to consider $A, B, C, b_{11}$, and $b_{12}$. Coincidentally, this gives us that part of $G$ available is the same as 4(a). We may convert this to the automaton in Fig. 6(a).

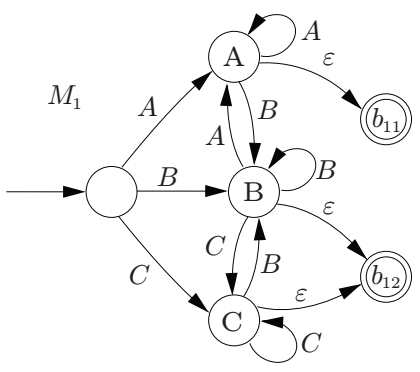

(a)

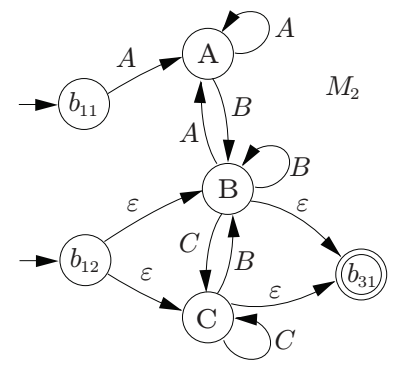

(b)
Fig. 6. a) The corresponding NFA when $b_{1}$ is the first sensor crossed. b) The NFA for consecutive sensor recordings $b_{1} b_{3}$.

The sensor that is activated next is $b_{3}$. Since agent $x$ must start from $b_{11}$ or $b_{12}$, the connectivity graph $G$ tells us that the agent can only pass $b_{3}$ from left to right. The part of $G$ available in this case is the same as 4(b). Follow similar construction, we obtain the corresponding automata shown in Fig. 6(b). Note that Fig. 6(b) represents two automata with one starts at from $b_{11}$ and one starts from $b_{12}$. The rest of the sensor recordings from $\mathbf{s}$ can be processed in the same manner. For an observation history s of length $m$, $m+1$ automata are obtained. Denote these automata $M_{1}$ through $M_{m+1}$. For implementation purpuse, we connect all of $M_{m+1}$ 's states to a single acceptance state $F$ via an $\epsilon$ transition. It is straightforward to observe that a story $\mathbf{p}$ is consistent with $\mathbf{s}$ if and only if the story string can be 
partitioned into pieces $P_{1}, \ldots, P_{m+1}$ such that $P_{i}$ is accepted by $M_{j}$. Alternatively, if we connect $M_{1}$ through $M_{m+1}$ together to get a composite automaton, $M$ (Fig. 7), then $\mathbf{p}$ must drive $M$ from start state to $F$.

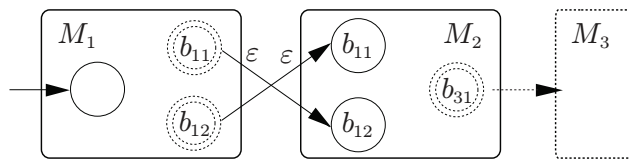

Fig. 7. Sketch of the composite automaton for the observation history string $b_{1} b_{3} O_{2} o_{2} b_{4}$.

With minimal effort, VALIDATEAGENTSTORY can be modified to work with the composite automaton $M$, which will be able to resolve Problem 1 and 2, keeping the dynamic programming framework intact. Although it does not make the algorithm more efficient, the approach provides an alternative interpretation of these problems: Searching $\mathbf{p}$ through $G_{s}$ now becomes simulating $M$ over p, making these problems tests of whether a string belongs to a regular language.

\section{Generalizations and Extensions}

For Problem 1 and 2, only a few stories are checked against an observation history $\mathbf{s}$. This is no longer the case for Problem 3 and 4 since an infinite number of possible stories must be checked in the process of solving these problems. This is where the automaton structure comes in: If we simulate a story $\mathbf{p}$ over an automaton, we know that on seeing $p_{i}$, there are a set of fixed states the automaton can be in. As long as we maintain these finite number of states, an infinite number of string patterns can be handled. In fact, existing results from Automata Theory have completed part of the job for us: Problem 3 and 4 can be viewed as the string edit distance problem between a string and an automaton (with the string being $\mathbf{p}$ and the automaton being $M$ ), for which efficient algorithms exist.

Our job is not done, however. Exisitng algorithms assume that the string $\mathbf{p}$ and the automaton $M$ are the inputs. In our problem, the automaton $M$ is an intermediate input built from $\mathbf{s}$ and $G_{s}$; thus, a direct adoption of approximate string matching algorithms may not fully explore the structure of $M$. In this section, we explore how the sequential nature of $M$ allows us to subdivide Problem 3 more effectively than off-the-shelf approaches. After solving Problem 3, we sketch at high level how the same structure helps solve Problem 4 as well.

\section{A. Partial Story}

We continue to work with the example from Fig. 2 and assume that the story is $\mathbf{p}=A B D E C$ and the observation history is $\mathbf{s}=b_{1} b_{2} O_{2} O_{2} b_{4}$. Suppose that we obtain automata $M_{j}$ 's as well as $M$ from s already. With the analysis from Section IV, Problem 3 becomes finding a shortest $\mathbf{p}^{\prime} \geq \mathbf{p}$ such that $\mathbf{p}^{\prime}$ is accepted by $M$. The problem may seem a bit daunting at first glance: An infinite number of $\mathbf{p}^{\prime}$ needs to be examined, as long as $\mathbf{p}^{\prime} \geq \mathbf{p}$.
However, any consistent $\mathbf{p}^{\prime}$ must drive the composite automaton $M$ to an accepting state. If we assume that some $\mathbf{p}^{\prime} \geq \mathbf{p}$ is one shortest such that is accepted by $M$, then it must have the format $\mathbf{p}^{\prime}=\omega_{1} A \omega_{2} B \omega_{3} D \omega_{4} E \omega_{5} C \omega_{6}$, in which $\omega_{i}$ 's denote the parts missing from p. Since $p_{1}=$ $A$, we search $M$ and find shortest string from the start state of $M$ to all copies of $A$ in $M$ (We may denote the copy of $A$ from $M_{j}$ as $(A, j)$ ); there are up to $m+1$ of these. Denoting these strings as $\sigma(1,0, j)$. For each $j$ there may be multiple such strings of the same shortest length; in this case any of these will do. By the principle of dynamic programming, $\left|\omega_{1}\right|+1=|\sigma(1,0, j)|$ for some $j$. Moving to $p_{2}=B$, for each $(B, k)$ let us denote a shortest string taking $M$ from some $(A, j)$ to $(B, k)$ as $\sigma(2, j, k)$. Among these $\sigma(2, j, k)$ 's for a fixed $k$, we need to get one such so that $\sigma(1,0, j)+\sigma(2, j, k)$ is a shortest. Again, the principle of dynamic programming tells us that for some $j, k$, $\left|\omega_{1}\right|+1=\mid \sigma(1,0, j)$ and $\left|\omega_{2}\right|+1=\sigma(2, j, k) \mid$. Following this method, if some simulation branches remain after all of $\mathbf{p}$ are exhausted, then a consistent $\mathbf{p}^{\prime}$ exists and one can be extracted via backtracking the search process.

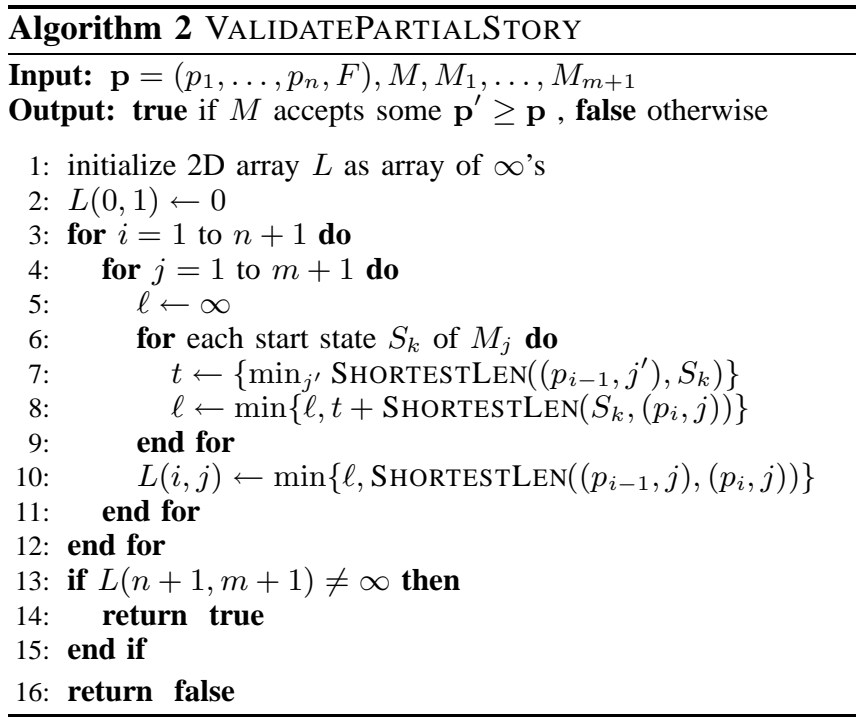

With above analysis, it becomes clear that the insight enabling the reduction of a factor of $m$ in VALIDATEAGENTSTORY also applies here. That is, in finding the shortest string containing $p_{1} \ldots p_{i}$ and taking $M$ from the start state to $\left(p_{i}, k\right)$, we do not need to look at $\left(p_{i-1}, j\right)$ for all $j \leq k$. Instead, we only need to know the shortest string that takes $\left(p_{i-1}, j\right), j \leq k-1$ for some $j$, to the start state(s) of $M_{k}$; the rest of the search is limited to $M_{k}$. Since searching inside $M_{k}$ for shortest path can be done with Dijkstra's algorithm in $O\left(n_{w}^{2}\right)$, the overall running time for the search part of validating a partial story is $O\left(n m n_{w}^{2}\right)$. The pesudocode is described in Algorithm 2. Note that we append to $\mathbf{p}$ an element $F$, which is the acceptance state of $M$. Element $L(i, j)$ of the 2D array $L$ stores the length of the shortest string that drives $M$ to the state $\left(p_{i}, j\right)$ and contains $p_{1} \ldots p_{i}$ as a subsequence. The subroutine $\operatorname{ShoRTEStLen}(a, b)$ returns the length of 
the shortest string that takes $M$ from state $a$ to state $b$. As discussed, we can obtain $\operatorname{ShorteStLEN}\left(\left(p_{i-1}, j^{\prime}\right), S_{k}\right)$ incrementally.

\section{B. Story with Errors}

Although Problem 4 appears harder than Problem 3, it is not clear that it is more time consuming. On one hand, to allow insertion, deletion, and substitution of story string p, we need to know the shortest edit distance to reach all states of $M$ for each $p_{i}$, instead of knowing only the shortest distance to states $\left(p_{i}, j\right)$. Denote this distance $D\left(p_{i}, T\right)$ in which $T$ is a state of $M$ and let $D\left(p_{i}, S, T\right)$ be the shortest edit distance from state $S$ (of $M$ ) to $T$ on $p_{i}$, we obtain the recursion

$$
D\left(p_{i}, T\right)=\min _{S}\left\{D\left(p_{i-1}, S\right)+D\left(p_{i}, S, T\right)\right\} .
$$

For a general automaton of $Q$ states, $D\left(p_{i}, S, T\right)$ requires $O\left(Q^{3}\right)\left(O\left(m^{3} m_{w}^{3}\right)\right.$ for our problem) computation time using a modified all pairs shortest path algorithm [30]. In our case, since $D\left(p_{i}, S, T\right)$ is $\infty$ for $S \in M_{k}, T \in M_{j}$ when $j<k$, we may subdivide the calculation of $D\left(p_{i}, T\right)$ further, staged at each $M_{j}$. Suppose $T$ is an internal state of $M_{j}$ (not start/end states), $\left\{S_{j}\right\}$ are the start states of $M_{j}$ (there are at most two of these), then the shortest edit distance from $S \in M_{j-1}$ to $T$, passing some $\left\{S_{j}\right\}$ can be obtained as

$$
\begin{aligned}
\min _{S}\left\{D\left(p_{i-1}, S\right)+\min \left\{\min _{S_{j}}\left\{D\left(p_{i}, S, S_{j}\right)+D\left(\epsilon, S_{j}, T\right)\right\},\right.\right. \\
\left.\left.\min _{S_{j}}\left\{D\left(\epsilon, S, S_{j}\right)+D\left(p_{i}, S_{j}, T\right)\right\}\right\}\right\} .
\end{aligned}
$$

We can regroup the formula as

$$
\begin{gathered}
\min _{S_{j}}\left\{\operatorname { m i n } \left\{\min _{S}\left\{D\left(p_{i-1}, S\right)+D\left(p_{i}, S, S_{j}\right)\right\}+D\left(\epsilon, S_{j}, T\right),\right.\right. \\
\left.\left.\min _{S}\left\{D\left(p_{i-1}, S\right)+D\left(\epsilon, S, S_{j}\right)\right\}+D\left(p_{i}, S_{j}, T\right)\right\}\right\} .
\end{gathered}
$$

Hence, instead of $O\left(\mathrm{~m}^{3} \mathrm{~m}_{w}^{3}\right)$ time, the sequential structure of $M$ enables us to cut the processing time per $p_{i}$ to at most $O\left(m m_{w}^{3}\right)$.

On the other hand, unlike in Problem 3, with the introduction of deletion and substitution, a matching story is always possible for Problem 4, as long as the sensor recordings are self-consistent (That is, the language of $M$ is not empty): In the worst case, we can change $\mathbf{p}$ to the shortest string accepted by $M$ via substitution, followed by insertion if $\mathbf{p}$ is too short and followed by deletion if $\mathbf{p}$ is too long. If we denote the length of the shortest string accepted by $M$ as $n^{\prime}$, then a $\mathbf{p}^{\prime}$, accepted by $M$ and closest to $\mathbf{p}$, cannot have length more than $\max \left\{n, n^{\prime}\right\}$. This is also the maximum number of edits necessary.

From above observation, we see that it is not necessary to carry all pairs shortest path search over $M$ for each $p_{i}$. This observation is the same one that enables the approach from [1] to keep only two levels of a transducer structure in memory. We adapt the same transducer construction to our problem, which has the high level structure illustrated in Fig. 8. Denote this transducer $U$. Comparing $U$ with that from [1], our transducer has additional structures: It is directed not only from top to bottom but also from left

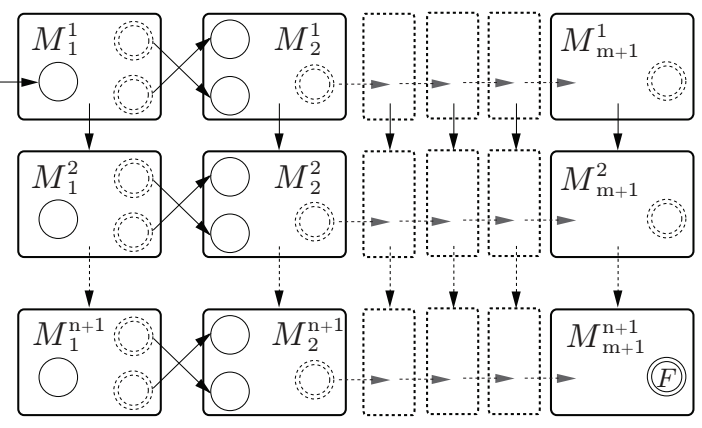

Fig. 8. Sketch of the transducer built from string $\mathbf{p}$ and automaton $M$.

to right. This means that searching $U$ can be partitioned into searching individual $M_{j}^{i}$,s from left to right then top to bottom. For Problem 3, finding $\mathbf{p}^{\prime}$ with smallest number of edits is equivalent to finding accepting string of $U$ with with the smallest cost. This is then a single source shortest path problem on $U$. As said, for each $i$, we carry out the search from left to right from $M_{1}^{i}$ to $M_{m+1}^{i}$, which takes time $O\left(m \cdot m_{w} \cdot m_{w} \lg m_{w}\right)$. Doing this for all $i$ (top to bottom) then takes time $O\left(n m m_{w}^{2} \lg m_{w}\right)$. In contrast, preprocessing along takes $O\left(m^{3} n_{w}^{4}\right)$ in [30] (Recall that $m, n$ are of comparable lengths). Our result is also slightly better than the (more general) algorithm presented in [1], which has time complexity $O\left(n m m_{w}^{2} \lg \left(m m_{w}\right)\right)$ in this context.

\section{Additional Extensions}

Having provided algorithms for Problems 2 through 4, many additional questions can be answered. We briefly discuss some of the possible extensions here.

Constraints. Besides the restrictions placed in Problem 1 through 4, many other problem variations are also possible and can be resolved similarly. The case that requires the suspect's story having a maximum error rate of $10 \%$ is one such constraint. As another example, the agent may be confident about certain parts of its story. In Problem 3, this translates to some substrings of the story string must be processed without inserting additional locations. Yet another example is that the story and/or the observation history may contain time "gaps", as an extension of Problem 2. These additional constraints can be dealt with by modifying the above algorithms.

Multiple agents. What if there are multiple agents in the workspace? As analyzed in [33], when an unknown number of agents are in the workspace, not all sensor recordings are triggered by agent $x$; there are $2^{|\mathbf{s}|}$ possible observation history by agent $x$. Moreover, the observation history has a different structure: The active occupancy sensors act as hubs that connect parts of the workspace together. Any agent can then pass an active occupancy sensor multiple times. Carrying over the analysis, we observe that the "unit automaton structure" still holds: A composite automaton can be built with individual automata having no more than $O\left(m_{w}\right)$ states. Such orthogonality allows direct combination of the multiple agent case from [33] with the solutions of 
Problems 2 through 4, generalizting them to include multiple agent cases.

\section{Vi. Conclusion And Future Directions}

In this paper, we studied the problem of validating an agent's story, which may be partial or contain errors, against the observation history of external sensors. In addition to deciding the validity of an agent's story in time linear in both the length of the story and the length of the observation history, our algorithms produce a path compatible with the sensor observations that is also closest to the agent's story in terms of string edit distance, whenever such a path exists.

Many intriguing questions remain. Focusing on the discrete setting, the next natural question appears to be the validation of multiple agents' combined story: It is possible that several agents' stories are valid when validated individually but problematic when put together. On the other hand, if we consider differential constraints for the agents, two immediate qualitative implications arise. First, since an agent's speed is limited, the exact time of sensor recordings, otherwise neglected, becomes relevant in filtering out long paths between sensor locations. Second, it becomes possible to measure how far away an agent's behavior deviates from the optimal behavior (In terms of distance traveled, for example). Besides these immediate extensions, another interesting direction is to study optimal sensor placements for the detective task, which was discussed to some extent in [23], [28]. Finally, we have only studied part of the spatial and temporal features of a story. With logic, it may become possible to interpret more challenging agent behavior such as "how" and "why".

Acknowledgments This work was supported in part by NSF grants 0904501 (IIS Robotics) and 1035345 (Cyberphysical Systems), DARPA SToMP grant HR0011-05-1-0008, and MURI/ONR grant N00014-09-1-1052.

\section{REFERENCES}

[1] C. Allauzen and M. Mohri. Linear-space computation of the editdistance between a string and a finite automaton. In London Algorithmics 2008: Theory and Practice, 2008.

[2] R. Alur, T. A. Henzinger, G. Lafferriere, and G. J. Pappas. Discrete abstractions of hybrid systems. In Proceedings of the IEEE, pages 971-984, 2000.

[3] J. Aslam, Z. Butler, F. Constantin, V. Crespi, G. Cybenko, and D. Rus. Tracking a moving object with a binary sensor network. pages 150 161. ACM Press, 2002.

[4] Y. Baryshnikov and R. Ghrist. Target enumeration via integration over planar sensor networks. In Proceedings of Robotics: Science and Systems IV, Zurich, Switzerland, June 2008.

[5] J. Castellanos, J. Montiel, J. Neira, and J. Tardós. The SPmap: A probabilistic framework for simultaneous localization and mapping. IEEE Transactions on Robotics \& Automation, 15(5):948-953, 1999.

[6] B. Chazelle. Approximation and decomposition of shapes. In J. T. Schwartz and C. K. Yap, editors, Algorithmic and Geometric Aspects of Robotics, pages 145-185. Lawrence Erlbaum Associates, Hillsdale, NJ, 1987.

[7] H. Choset and K. Nagatani. Topological simultaneous localization and mapping (T-SLAM). IEEE Transactions on Robotics \& Automation, 17(2):125-137, 2001.

[8] D. C. Conner, H. Kress-gazit, H. Choset, A. A. Rizzi, and G. Pappas. Valet parking without a valet. In IEEE/RSJ International Conference on Intelligent Robots \& Systems, 2007.
[9] M. Dehn. Papers on Group Theory and Topology. Springer-Verlag, Berlin, 1987.

[10] E. W. Dijkstra. A note on two problems in connexion with graphs. Numerische Mathematik, 1:269-271, 1959.

[11] G. Dissanayake, P. Newman, S. Clark, H. F. Durrant-Whyte, and M. Csorba. A solution to the simultaneous localisation and map building (SLAM) problem. IEEE Transactions on Robotics \& Automation, 17(3):229-241, 2001.

[12] D. B. A. Epstein, M. S. Paterson, G. W. Camon, D. F. Holt, S. V. Levy, , and W. P. Thurston. Word Processing in Groups. A. K. Peters, Natick, MA, 1992.

[13] Q. Fang, F. Zhao, and L. Guibas. Counting targets: Building and managing aggregates in wireless sensor networks. Technical Report P2002-10298, Palo Alto Research Center, June 2002.

[14] W. Kim, K. Mechitov, J. Choi, and S. Ham. On target tracking with binary proximity sensors. In ACM/IEEE International Conference on Information Processing in Sensor Networks, pages 301-308. IEEE Press, 2005.

[15] H. Kress-gazit, G. E. Fainekos, and G. Pappas. Wheres waldo? sensor-based temporal logic motion planning. In Proceedings IEEE International Conference on Robotics \& Automation, pages 31163121, 2007.

[16] S. M. LaValle. Planning Algorithms. Cambridge University Press, Cambridge, U.K., 2006. Also available at http://planning.cs.uiuc.edu/.

[17] S. M. LaValle. Filtering and planning in information spaces. Technical report, Dept. of Comp. Sci., University of Illinois, Oct 2009.

[18] M. Montemerlo, S. Thrun, D. Koller, and B. Wegbreit. FastSLAM: A factored solution to the simultaneous localization and mapping problem. In Proceedings AAAI National Conference on Artificial Intelligence, 1999.

[19] E. W. Myers and W. Miller. Approximate matching of regular expressions. Bulletin of Mathematical Biology, 51(1):5-37, 1989.

[20] G. Navarro. A guided tour to approximate string matching. ACM Computing Surveys, 33(1):31-88, 2001.

[21] R. Parr and A. Eliazar. DP-SLAM: Fast, robust simultaneous localization and mapping without predetermined landmarks. In Proceedings International Joint Conference on Artificial Intelligence, 2003.

[22] N. Piterman, A. Pnueli, and Y. Sa'ar. Synthesis of reactive(1) designs. In Proceedings Verification, Model Checking, and Abstract Interpretation, pages 364-380. Springer, 2006.

[23] B. S. Y. Rao, H. F. Durrant-Whyte, and J. S. Sheen. A fully decentralized multi-sensor system for tracking and surveillance. International Journal of Robotics Research, 12(1):20-44, February 1993.

[24] D. Sankoff and J. B. Kruskal. Time Wraps, String Edits and Macromolecules: The Theory and Practice of Sequence Comparison. Addison-Wesley, Reading, MA, 1983.

[25] N. Shrivastava, R. Mudumbai, U. Madhow, and S. Suri. Target tracking with binary proximity sensors: fundamental limits, minimal descriptions, and algorithms. In Proc. 4th Internat. Conf. on Embedded Networked Sensor Systems, 2006, pages 251-264. ACM Press, 2006.

[26] J. Singh, R. Kumar, U. Madhow, S. Suri, and R. Cagley. Tracking multiple targets using binary proximity sensors. In Proc. Information Processing in Sensor Networks, 2007.

[27] S. Thrun, W. Burgard, and D. Fox. A probabilistic approach to concurrent mapping and localization for mobile robots. Machine Learning, 31(5):1-25, April 1998.

[28] B. Tovar, F. Cohen, and S. M. LaValle. Sensor beams, obstacles, and possible paths. In Proceedings Workshop on Algorithmic Foundations of Robotics, 2008.

[29] B. Tovar, R Murrieta, and S. M. LaValle. Distance-optimal navigation in an unknown environment without sensing distances. IEEE Transactions on Robotics, 23(3):506-518, June 2007.

[30] R. A. Wagner. Order-n correction for regular languages. Communications of the ACM, 17(5):265-268, 1974.

[31] R. A. Wagner and J. I. Seiferas. Correcting counter-automatonrecognizable languages. SIAM Journal on Computing, 7(3):357-375, August 1978.

[32] J. Yu and S. M. LaValle. Tracking hidden agents through shadow information spaces. In Proceedings IEEE International Conference on Robotics \& Automation, 2008.

[33] J. Yu and S. M. LaValle. Cyber detectives: Determining when robots or people misbehave. In Proceedings Workshop on Algorithmic Foundations of Robotics, 2010. 